\title{
Pharmacotherapy Initiation
}

National Cancer Institute

\section{Source}

National Cancer Institute. Pharmacotherapy Initiation. NCI Thesaurus. Code C128530.

The start of treatment with a drug; the first prescription or order for a drug associated with a given course of treatment. 